\title{
First histopathological evidence of irreversible pulmonary vascular disease in dasatinib-induced pulmonary arterial hypertension
}

\author{
To the Editor:
}

We read with interest the article by WeATHERALD et al. [1] on the long-term outcomes of pulmonary arterial hypertension (PAH) induced by dasatinib. The authors reported 21 incident cases of PAH confirmed by right heart catheterisation (RHC) and associated with dasatinib. Although a majority of patients improved after dasatinib discontinuation, PAH persisted in over one-third of cases during long-term follow-up and two additional patients had persistent exercise pulmonary hypertension despite normalisation of resting haemodynamic measures. Similarly, SHAH et al. [2] reported 41 cases of dasatinib-induced PAH confirmed by RHC with complete resolution of PAH in only $58 \%$ of patients, provided that follow-up RHC or echocardiography was most often not documented. As mentioned by WEATHERALD et al. [1], these data suggest that dasatinib is likely to cause irreversible pulmonary vascular dysfunction and remodelling. An experimental model in rats and human pulmonary endothelial cells supports this hypothesis as it showed that dasatinib causes dose-dependent pulmonary endothelial dysfunction and apoptosis through the production of mitochondrial reactive oxygen species, a phenomenon that was not observed with imatinib [3]. To date, there is no published evidence of such pathological abnormalities in the human lung. We report the case of a patient who developed dasatinib-induced severe $\mathrm{PAH}$ that progressed in spite of drug cessation and aggressive $\mathrm{PAH}$-specific therapy and ultimately underwent lung transplantation.

A 32-year old male was diagnosed with chronic myeloid leukaemia (BCR-ABL1+) which was treated sequentially with imatinib, nilotinib and dasatinib. 36 months after dasatinib initiation, he developed pre-capillary pulmonary hypertension confirmed by RHC with mean pulmonary arterial pressure (mPAP) of $57 \mathrm{mmHg}$, pulmonary artery wedge pressure of $9 \mathrm{mmHg}$, cardiac index of $1.4 \mathrm{~L} \cdot \mathrm{min}^{-1} \cdot \mathrm{m}^{-2}$ and pulmonary vascular resistance (PVR) of 18.5 Wood units (WU), non-reactive to inhaled iloprost. Except for the above-mentioned tyrosine kinase inhibitors (TKIs), the patient had not been previously exposed to any chemotherapeutic agent or anorexigen drug. A comprehensive workup, including echocardiography, contrast-enhanced computed tomography (CT) of the chest, ventilation/perfusion lung scan, abdominal ultrasound, autoimmunity screening and HIV serology, excluded other causes of pulmonary hypertension, which thus was attributed to dasatinib. At the time of PAH diagnosis, the patient presented with New York Heart Association (NYHA) functional class III and 6-min walk distance (6MWD) was $340 \mathrm{~m}$. Chest CT and echocardiography showed significant bilateral pleural effusions and a minor pericardial effusion. Following discontinuation of dasatinib and the introduction of PAH treatment, a combination of phosphodiesterase type 5 (PDE-5) inhibitor and an endothelin receptor antagonist (ERA), NYHA functional class improved to class I/II and 6MWD increased to $610 \mathrm{~m}$ within 12 months. A follow-up RHC performed 10 months after PAH diagnosis confirmed significant haemodynamic improvement with $\mathrm{mPAP}$ decrease to $38 \mathrm{mmHg}$, cardiac index increase to $5.8 \mathrm{~L} \cdot \mathrm{min}^{-1} \cdot \mathrm{m}^{-2}$ and near-normalisation of PVR to $2.8 \mathrm{WU}$.

Owing to molecular relapse of chronic myeloid leukaemia, nilotinib was reintroduced at reduced dose ( $400 \mathrm{mg}$ per day) by considering that it was less likely than dasatinib to induce PAH. During the 3 years

@ERSpublications

The presence of irreversible pulmonary vascular lesions explains why dasatinib-induced PAH can frequently persist http://ow.ly/MKwI30hRwfc

Cite this article as: Daccord C, Letovanec I, Yerly P, et al. First histopathological evidence of irreversible pulmonary vascular disease in dasatinib-induced pulmonary arterial hypertension. Eur Respir J 2018; 51: 1701694 [https://doi.org/10.1183/13993003.01694-2017]. 
following nilotinib resumption and despite further dose reduction to $200 \mathrm{mg}$ per day, we observed a gradual clinical and functional worsening, suggesting PAH progression. RHC was repeated and showed significant aggravation of $\mathrm{PAH}$ with an increase of $\mathrm{mPAP}$ to $70 \mathrm{mmHg}$, a drop of cardiac index to 2.2 $\mathrm{L} \cdot \mathrm{min}^{-1} \cdot \mathrm{m}^{-2}$, a severe rise in PVR to $11.8 \mathrm{WU}$, as well as right atrial pressure above $14 \mathrm{mmHg}$ and low mixed venous oxygen saturation as factors of poor prognosis. $\mathrm{PAH}$ therapy was intensified with continuous intravenous treprostinil infusion up to $40 \mathrm{ng} \cdot \mathrm{kg}^{-1} \cdot \mathrm{min}^{-1}$, in addition to maximal oral doses of PDE-5 inhibitor (sildenafil) and ERA (ambrisentan). However, PAH further progressed and over the following 6 months, the patient developed symptomatic right heart failure with NYHA functional class IV, exertional presyncopes, refractory hydrosodic retention and severe hypoxaemia that ultimately necessitated
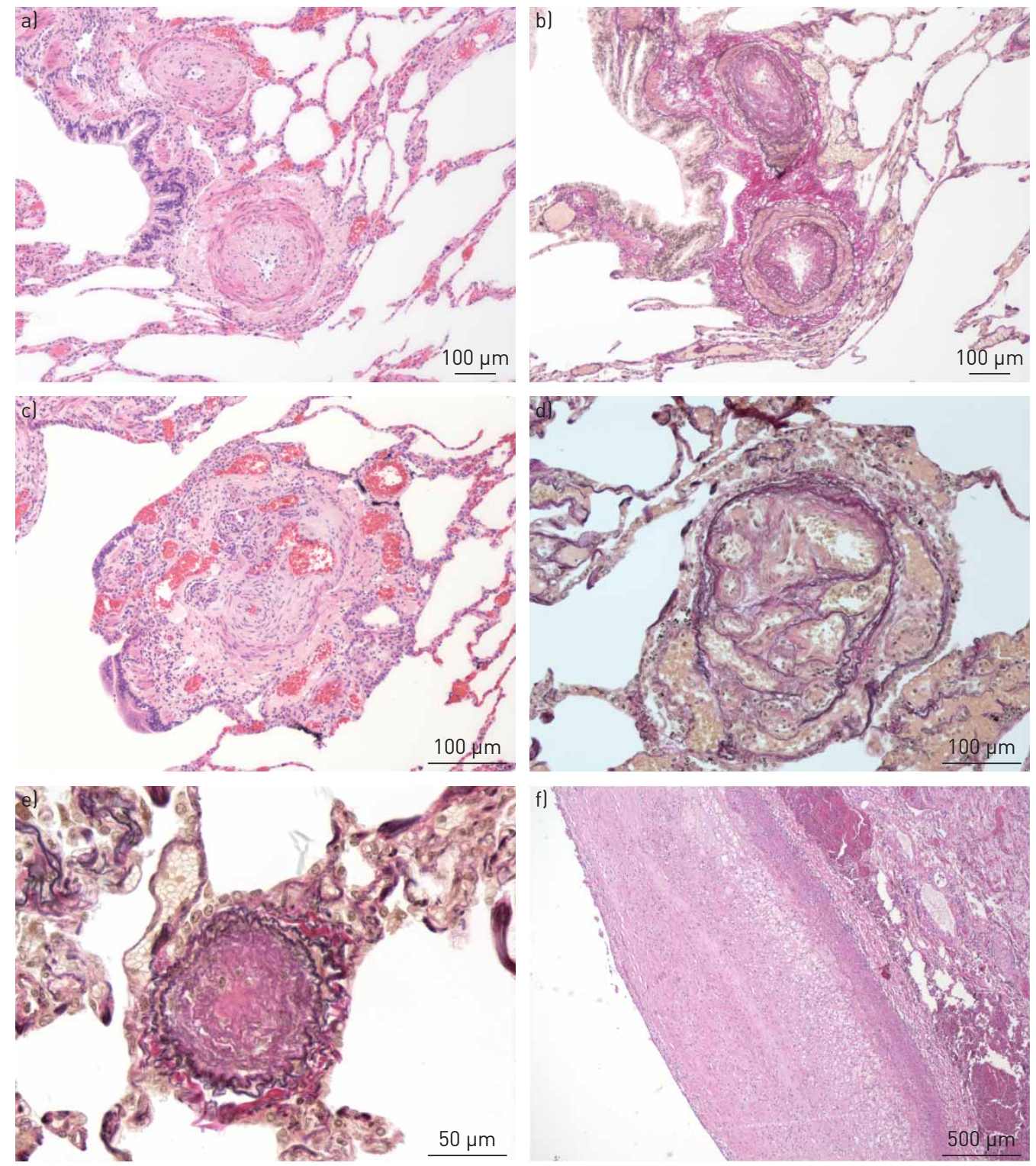

FIGURE 1 Histopathological features of dasatinib-induced pulmonary arteriopathy observed on explanted lungs: a) small pulmonary artery with medial hypertrophy and concentric non-laminar intimal thickening, haematoxylin eosin (HE) stain. b) Same lesion as (a) with van Gieson Elastin (VGE) stain for elastin highlighting the internal and external elastic laminae of the arterial wall. c) Plexiform lesion corresponding to a complex vascular structure originating from remodelled pulmonary arteries and formed by focal proliferation of endothelial cells, HE stain. d) Same lesion as (c) with VGE stain showing disruption of the elastic laminae associated with partial destruction and remodelling of the arterial walls. e) Pulmonary arteriole beneath $70 \mu \mathrm{m}$ showing complete obliteration through mainly intimal thickening, VGE stain. Note: changes in small arterioles were heterogeneously distributed throughout the lung. f) Proximal pulmonary artery showing important atherosclerosis, HE stain. Note: neither bronchial artery remodelling (hypertrophy/ dilatation) nor pulmonary vein remodelling (smooth muscle hyperplasia and/or intimal fibrosis) were observed. 
continuous intravenous diuretics, recurrent thoracenteses, high flow oxygen therapy and haemodynamic support with noradrenaline and levosimendan infusions. In view of an immediately life-threatening situation due to $\mathrm{PAH}$ and given the favourable prognosis of chronic myeloid leukaemia on long-term TKI, it was decided, after multidisciplinary discussion, to perform bilateral lung transplantation which was successfully achieved under perioperative veno-arterial extracorporeal membrane oxygenation support, after 20 days on the waiting list. The patient was rapidly weaned off the haemodynamic support and extubated after 3 days. A transoesophageal echocardiography performed $24 \mathrm{~h}$ after surgery showed normalisation of PAP and resolution of right ventricular dilatation with only minor residual dysfunction. After satisfactory cicatrisation of bronchial anastomoses and in the absence of imatinib resistance, imatinib $400 \mathrm{mg}$ per day was reintroduced. 6 months after lung transplantation, chronic myeloid leukaemia is currently controlled on imatinib with undetectable BCR-ABL transcript level (major molecular response). No mutation in the BMPR2 gene was found.

The analysis of the explanted lungs showed atherosclerosis and typical histopathological features of chronic PAH [4]. Pulmonary arteriopathy was characterised by major vascular wall remodelling with medial hypertrophy and concentric laminar intimal thickening, as well as plexiform lesions (figure 1), without evidence of pulmonary veno-occlusive or thromboembolic disease.

In our opinion, this case deserves attention as it suggests a possible severe evolution of dasatinib-induced $\mathrm{PAH}$ despite transition to nilotinib and maximal PAH-specific therapy. To our knowledge, there are no published data regarding lung transplantation in such a context. Moreover, this is the first case providing evidence of dasatinib-induced characteristic pulmonary arterial remodelling in humans, as seen in other causes of PAH. It appears to confirm the hypothesis raised by WEATHERALD et al. [1] of irreversible pulmonary vascular disease in patients with persistent $\mathrm{PAH}$ in spite of dasatinib discontinuation. It could also explain why, even though clinical and functional improvement is most often observed upon treatment discontinuation, abnormal haemodynamic can persist. In our case, it is interesting to note that PAH initially improved after dasatinib cessation and with dual PAH therapy, with near-normalisation of PVR, and worsened only after nilotinib reintroduction, raising questions about the role of nilotinib in PAH progression. However, except for one case of suspected nilotinib-induced PAH without confirmation of diagnosis by RHC [5], there is currently no confirmed case of incident PAH caused by nilotinib, nor reported case of PAH worsening or relapse following replacement of dasatinib by nilotinib, although nilotinib is the most frequently used TKI after dasatinib withdrawal [6]. On the other hand, there have been published cases of incident or worsening PAH related to ponatinib or bosutinib in patients with previously documented dasatinib-induced pleural effusions or PAH [7-9]. Consequently, we cannot exclude that nilotinib, similarly to ponatinib and bosutinib, played some role in PAH progression and perpetuated pulmonary vascular toxicity following previous injury by dasatinib. Alternatively, the disease might have inevitably progressed, as seen in other forms of $\mathrm{PAH}$, despite dasatinib withdrawal and after transient improvement on PAH therapy.

The risk of PAH recurrence after lung transplantation and reintroduction of TKIs is unknown but seems unlikely as, on the one hand, it now depends on the predisposition of the pulmonary graft to PAH, and on the other hand, treatment with imatinib is not known to cause PAH and has even showed some beneficial effects in patients with PAH [10]. In case of resistance or intolerance to imatinib, rechallenge with dasatinib would be contraindicated. In the absence of previous graft exposure to dasatinib, the replacement of imatinib by nilotinib could be an option to be considered cautiously and with close monitoring.

In conclusion, this extreme case illustrates the possible occurrence of irreversible pulmonary vascular remodelling and suggests that dasatinib induced PAH. It is difficult to implicate dasatinib conclusively as the sole agent responsible for the development of $\mathrm{PAH}$ in this case or in patients in the other reports. However, given the number of cases associated with dasatinib, it should be considered as a major factor in the development of PAH in chronic myeloid leukaemia patients.

Cécile Daccord $^{1}$, Igor Letovanec ${ }^{2}$, Patrick Yerly ${ }^{3}$, Jonathan Bloch $^{4}$, Adam Ogna ${ }^{1}{ }^{1}$, Laurent P. Nicod ${ }^{1,5}$ and John-David Aubert $\mathbb{( i )}^{1,5}$

${ }^{1}$ Dept of Respiratory Medicine, Lausanne University Hospital, Lausanne, Switzerland. ${ }^{2}$ Dept of Pathology, Lausanne University Hospital, Lausanne, Switzerland. ${ }^{3}$ Dept of Cardiology, Lausanne University Hospital, Lausanne, Switzerland. ${ }^{4}$ Service and Central Laboratory of Hematology, Oncology Dept, Lausanne University Hospital, Lausanne, Switzerland. ${ }^{5}$ Lung Transplantation Unit, Lausanne University Hospital, Lausanne, Switzerland.

Correspondence: John-David Aubert, Dept of Respiratory Medicine, Lausanne University Hospital (CHUV), Rue du Bugnon 46, 1011 Lausanne, Switzerland. E-mail: john-david.aubert@chuv.ch

Received: Aug 192017 | Accepted after revision: Dec 312017

Conflict of interest: None declared. 


\section{References}

1 Weatherald J, Chaumais M-C, Savale L, et al. Long-term outcomes of dasatinib-induced pulmonary arterial hypertension: a population-based study. Eur Respir J 2017; 50: 1700217.

2 Shah NP, Wallis N, Farber HW, et al. Clinical features of pulmonary arterial hypertension in patients receiving dasatinib. Am J Hematol 2015; 90: 1060-1064.

3 Guignabert C, Phan C, Seferian A, et al. Dasatinib induces lung vascular toxicity and predisposes to pulmonary hypertension. J Clin Invest 2016; 126: 3207-3218.

4 Pietra GG, Capron F, Stewart S, et al. Pathologic assessment of vasculopathies in pulmonary hypertension. J Am Coll Cardiol 2004; 43: 25S-32S.

5 Zakrzewski D, Seferynska I, Warzocha K, et al. Elevation of pulmonary artery pressure as a complication of nilotinib therapy for chronic myeloid leukemia. Int J Hematol 2012; 96: 132-135.

6 Montani D, Bergot E, Günther S, et al. Pulmonary arterial hypertension in patients treated by dasatinib. Circulation 2012; 125: 2128-2137.

7 Quilot FM, Georges M, Favrolt N, et al. Pulmonary hypertension associated with ponatinib therapy. Eur Respir J 2016; 47: 676-679.

8 Hickey PM, Thompson AA, Charalampopoulos A, et al. Bosutinib therapy resulting in severe deterioration of pre-existing pulmonary arterial hypertension. Eur Respir J 2016; 48: 1514-1516.

9 Riou M, Seferian A, Savale L, et al. Deterioration of pulmonary hypertension and pleural effusion with bosutinib following dasatinib lung toxicity. Eur Respir J 2016; 48: 1517-1519.

10 Hoeper MM, Barst RJ, Bourge RC, et al. Imatinib mesylate as add-on therapy for pulmonary arterial hypertension: results of the randomized IMPRES study. Circulation 2013; 127: 1128-1138. 